Pacific Journal of Mathematic 


\title{
ON CHARACTERISTIC FUNCTIONS OF BANACH SPACE VALUED RANDOM VARIABLES
}

\author{
R. K. GETOOR
}

1. Introduction. In recent years several authors have considered the notion of random variables with values in a Banach space, $\mathfrak{X}$. One of the basic problems is to characterize those positive definite functions on $\mathfrak{X}^{*}$ that are characteristic functions of such random variables. Mourier [4] has given a solution to this problem if $\mathfrak{X}$ is separable and reflexive. The purpose of this paper is to give another solution of this problem. Our results are valid if $\mathfrak{X}$ is reflexive. However the contribution of this paper is not so much the removal of the condition of separability, rather we feel that our method sheds new light on the problem and aids in understanding it. The basic tool that we use is the concept of a weak distribution as introduced by Segal [5], and this idea succeeds in unifying the theory.

Section 2 contains the basic definitions and preliminaries. The main results are contained in $\S 3$ but in a form slightly more general than needed for the problem at hand. However we will need the results in this generality in a future paper. The contents of $\S 3$ are clearly valid in any locally convex linear topological space. Finally in $\S 4$ our solution to the problem stated above is given along with some examples and consequences.

The considerations of Bochner in chapters five and six of [1] are somewhat related to our problem.

2. Definitions. Let $(\Omega, \widetilde{F}, P)$ be a probability space, that is, $\Omega$ is an abstract point set, $\widetilde{F}$ a $\sigma$-algebra of subsets of $\Omega$, and $P$ is a measure on $(\Omega, \widetilde{F})$ with $P(\Omega)=1$. Let $\mathfrak{X}$ be a real Banach space ${ }^{1}$ and $\mathfrak{X}^{*}$ its conjugate space. Let $X: \Omega \rightarrow \mathfrak{X}$, we will call $X$ an $\mathfrak{X}$ valued random variable if $X$ is weakly measurable, that is, if $\left\langle x^{*}, X(\omega)\right\rangle$ is a real valued $\mathfrak{F}$ measurable function for each $x^{*} \in \mathfrak{X}^{*}$. Let $E(X)$ be the Pettis integral of $X$ with respect to $P$, provided it exists. Thus $E(X)$ is the unique element of $\mathfrak{X}$ such that $\left\langle x^{*}, E(X)\right\rangle=E\left\{\left\langle x^{*}, X\right\rangle\right\}=\int\left\langle x^{*}, X(\omega)\right\rangle d P$ for each $x^{*} \in \mathfrak{X}^{*}$. The characteristic function of $X$ is defined as follows,

$$
\phi\left(x^{*}\right)=E\left\{e^{i\left\langle x^{*}, X\right\rangle}\right\}=\int e^{i \lambda} d F\left(\left(x^{*} ; \lambda\right)\right.
$$

Received January 16, 1956. This research supported in part by Office of Ordnance Research, U.S. Army, contract DA-36-034-ORD-1296 RD.

1 The extension to a complex Banach space is essentially clear. 
where $F\left(x^{*} ; \lambda\right)$ is the distribution function of the real valued random variable $\left\langle x^{*}, X(w)\right\rangle$. It follows that $\phi(0)=1, \phi$ is positive definite, and $\phi$ is continuous. For a detailed discussion of the above concepts see [4].

If we put $L\left(x^{*}\right)=\left\langle x^{*}, X(\omega)\right\rangle$ then $L$ is a linear map from $\mathfrak{X}^{*}$ to random variables. Segal [5] defines a "weak distribution", $L$, on $\mathfrak{X}$ to be a linear map from $\mathfrak{X}^{*}$ to random variables. However there are two interpretations of this statement. We may mean $L\left(\sum_{i=1}^{n} a_{i} x_{i}^{*}\right)=\sum_{i=1}^{n} a_{i} L\left(x_{i}^{*}\right)$ with probability one or the stronger statement that for almost all $\omega$ the function $L(\cdot, \omega)$ is linear ${ }^{2}$. Theorem 2 of the next section shows that these two possibilities are actually equivalent. Thus since there is a possible ambiguity and since we want to consider a weak distribution as the generalization of an ordinary $n$-dimensional distribution we make the following definition.

DEFINITION 2.1. A weak distribution, $L$, on $\mathfrak{X}$ is a map which assigns to each finite collection of elements $\left(x_{1}^{*}, \cdots, x_{n}^{*}\right)$ in $\mathfrak{X}^{*}$ an $n$-dimensional distribution function $F_{n}\left(x_{1}^{*}, \lambda_{1} ; \cdots ; x_{n}^{*}, \lambda_{n}\right)$ such that

(1) $F_{n}$ is symmetric in the pairs $\left(x_{i}^{*}, \lambda_{i}\right)$.

(2) $F_{n}\left(x_{1}^{*}, \lambda_{1} ; \cdots ; x_{n}^{*}, \infty\right)=F_{n-1}\left(x_{1}^{*}, \lambda_{1} ; \cdots ; x_{n-1}, \lambda_{n-1}\right)$.

(3) If $\sum_{i=1}^{n} a_{1} x_{i}^{*}=0$ then

$$
\int_{\sum_{i=1}^{n} a_{i} \lambda_{i} \leqq \lambda} d F_{n}\left(x_{1}^{*}, \lambda_{1} ; \cdots ; x_{n}^{*}, \lambda_{n}\right)=\varepsilon(\lambda)
$$

where $\varepsilon(\lambda)$ is the unit distribution, $\varepsilon(\lambda)=\left\{\begin{array}{ll}0 & \lambda<0 \\ 1 & \lambda \geq 0\end{array}\right.$.

Note. Condition (3) implies that if $x^{*}=\sum_{i=1}^{n} a_{i} x_{i}^{*}$ then

$$
F\left(x^{*}, \lambda\right)=\int_{\sum_{i=1}^{n} a_{i} \lambda_{i} \leqq \lambda} d F_{n}\left(x_{1}^{*}, \lambda_{1} ; \cdots ; x_{n}^{*}, \lambda_{n}\right) .
$$

ExAMPLE. If $X$ is an $\mathfrak{X}$ valued random variable then there is associated with it in a natural way a weak distribution which assigns to $\left(x_{1}^{*}, \cdots, x_{n}^{*}\right)$ the joint distribution function of the random variables $\left\langle x_{i}^{*}, X(\omega)\right\rangle$, thus,

$$
F\left(x_{1}^{*}, \lambda_{1} ; \cdots ; x_{n}^{*}, \lambda_{n}\right)=\operatorname{Pr}\left[\left\langle x_{i}^{*}, X(\omega)\right\rangle \leqq \lambda_{i}, i=1, \cdots, n\right] .
$$

"Since "random variable" in [5] in treated as a residue class module null sets, it clearly seems that the definition of weak distribution given there refers to the first interpretation. 
Given a weak distribution $L$ we define its characteristic function $\phi\left(x^{*}\right)$ by

$$
\phi\left(x^{*}\right)=\int e^{i \lambda} d F\left(x^{*} ; \lambda\right) .
$$

It is clear from (2.1) that if $L$ comes from $X$ (as in the example) then the characteristic function of $L$ and $X$ are the same.

We are going to give conditions that a weak distribution come from some $\mathfrak{X}$ valued random variable and hence that $\phi$ be the characteristic function of some $\mathfrak{X}$ valued random variable.

3. The main theorems. From the definitions in the preceding section we see that $L$ and its characteristic fuunction $\phi$ are both defined relative to $\mathfrak{X}^{*}$. In other words in the study of the relations between $\phi$ and $L$ the space $\mathfrak{X}$ plays no role. We are thus led to define a $q$-weak distribution on $\mathfrak{X}$ as a map, $L$, from finite sets of elements $\left(x_{1}, \cdots, x_{n}\right)$ in $\mathfrak{X}$ to distribution functions which satisfies the conditions of Definition 2.1. We can now state our first theorem.

THEOREM 1. There is a unique one-to-one correspondence between $q$ weak distributions $L$ defined on $\mathfrak{X}$ and positive definite functions $\phi$ defined on $\mathfrak{X}$ satisfying (i) $\phi(0)=1$ (ii) $\phi$ is continuous on each finite dimensional subspace of $\mathfrak{X}$. We say that $\phi$ is the Fourier transform of $L$ and denote the correspondence by $\phi=\mathfrak{F}(L) .^{3}$

Proof. In the following we will need a formula for change of variables in Lebesgue-Stieltjes integrals that we give here for convenience. If

$$
F(\lambda)=\int_{\sum_{i=1}^{n} \alpha_{i} \lambda_{i} \leq \lambda} d F_{n}\left(\lambda_{1}, \cdots, \lambda_{n}\right)
$$

then for any bounded Borel measurable function, $f$, we have

$$
\int f(\lambda) d F(\lambda)=\int f\left(\sum_{i=1}^{n} a_{i} \lambda_{i}\right) d F_{n}\left(\lambda_{1}, \cdots, \lambda_{n}\right) .
$$

Given $L$ we define the corresponding $\phi$ by

$$
\phi(x)=\int e^{i \lambda} d F(x ; \lambda)
$$

3 This result was essentially contained in a lecture of I. Segal given at the Institute for Advanced Study during the academic year 1954-55. See also [1]. Note that (ii) can be replaced by the equivalent condition that $\phi$ is continuous at 0 on each finite dimensional subspace. 
Clearly $\phi(0)=1$ and since

$$
\phi\left(\sum_{k=1}^{n} \alpha_{k} x_{k}\right)=\int e^{i \lambda} d F\left(\sum_{k=1}^{n} \alpha_{k} x_{k} ; \lambda\right)=\int e^{i \sum_{k=1}^{n} \alpha_{k} \lambda_{k}} d F_{n}\left(x_{1}, \lambda_{1} ; \cdots ; x_{n}, \lambda_{n}\right)
$$

(using the formula stated above) it is evident that $\phi$ is continuous on each finite dimensional subspace of $\mathfrak{X}$. Moreover

$$
\begin{aligned}
\sum_{j, k=1}^{n} \alpha_{k} \bar{\alpha}_{j} \phi\left(x_{k}-x_{j}\right) & =\sum_{j, k=1}^{n} \alpha_{k} \bar{\alpha}_{j} \int e^{i \lambda} d F\left(x_{k}-x_{j}, \lambda\right) \\
& =\sum_{j, k=1}^{n} \alpha_{k} \bar{\alpha}_{j} \int e^{i\left(\lambda_{k}-\lambda_{j}\right)} d F_{2}\left(x_{k}, \lambda_{k} ; x_{j}, \lambda_{j}\right) \\
& =\sum_{j, k=1}^{n} \alpha_{k} \bar{\alpha}_{j} \int e^{i\left(\lambda_{k}-\lambda_{j}\right)} d F_{n}\left(x_{1}, \lambda_{1} ; \cdots ; x_{n}, \lambda_{n}\right) \\
& =\int\left|\sum_{j, k=1}^{n} \alpha_{k} e^{i \lambda_{k}}\right|^{2} d F_{n} \geqq 0 .
\end{aligned}
$$

Thus $\phi$ is positive definite and satisfies the conditions of Theorem 1 .

Conversely suppose we are given $\phi$ satisfying the conditions of Theorem 1. For any finite set of elements $\left(x_{1}, \cdots, x_{n}\right)$ we consider the function $\psi\left(\alpha_{1}, \cdots, \alpha_{n}\right)=\phi\left(\sum_{k=1}^{n} \alpha_{k} x_{k}\right)$. It then follows that $\psi$ is an $n$ dimensional characteristic function in the ordinary sense. Hence by the $n$-dimensional Bochner theorem there exists a distribution function $F_{n}\left(x_{1}, \lambda_{1} ; \cdots ; x_{n}, \lambda_{n}\right)$ such that

$$
\phi\left(\sum_{k=1}^{n} \alpha_{k} x_{k}\right)=\psi\left(\alpha_{1}, \cdots, \alpha_{n}\right)=\int e^{i \sum_{k=1}^{n} \alpha_{k} \lambda_{k}} d F_{n}\left(x_{1}, \lambda_{1} ; \cdots ; x_{n}, \lambda_{n}\right) .
$$

By using the uniqueness assertion of the $n$-dimensional Bochner theorem it is easy to show that the above construction actually defines a $q$-weak distribution on $\mathfrak{X}$. The fact that the correspondence established between the $\phi$ 's and the $L$ 's is one-to-one (and unique) again follows from the uniqueness in the $n$-dimensional Bochner theorem.

CoROLlary 1. A necessary and sufficient condition that $\phi$ be continuous on $\mathfrak{X}$ is that $F(x, \lambda) \rightarrow \varepsilon(\lambda)$ as $x \rightarrow 0$.

Proof. This is an immediate consequence of the representation (3.2) and the properties of ordinary characteristic functions.

The following example shows that there actually exist positive definite functions continuous on each finite dimensional subspace without being continuous. Let $\mathfrak{X}$ be a separable Hilbert space and let $\left\{e_{\sigma}\right\}$ be a linear base, thus if $x \in \mathfrak{X}$ then $x=\sum_{1}^{n} \alpha_{j} e_{\sigma_{j}}$ and this expression is unique. It is no restriction to assume $\left\|e_{\sigma}\right\|=1$ for all $\sigma$. Let $\left\{\sigma_{n}\right\}$ be a given 
sequence from $\{\sigma\}$, and let $Y_{n}$ be independent Gaussian random variables (real valued) such that $E\left(Y_{n}\right)=0$ and $E\left(Y_{n}^{2}\right)=n$. Put $Y_{\sigma_{n}}=Y_{n}$ and $Y_{\sigma}=0$ is $\sigma \neq \sigma_{n}$ for some $n$. If $x=\sum_{1}^{k} \alpha_{j} e_{\sigma_{j}}$ we define $L(x)=\sum_{1}^{k} \alpha_{j} Y_{\sigma_{j}}$. This then defines a $q$-weak distribution, $L$, on $\mathfrak{X}$ as described in the example of $\S 2$, that is, $F_{n}\left(x_{1}, \lambda_{1} ; \cdots ; x_{n}, \lambda_{n}\right)$ is the joint distribution of $L\left(x_{1}\right)$, $\cdots, L\left(x_{n}\right)$. Let $\phi=\mathfrak{F}(L)$ then, according to Theorem $2, \phi$ is continuous on each finite dimensional subspace. However $\frac{1}{\sqrt{n}} e_{\sigma_{n}} \rightarrow 0$ while for each $n F\left(\frac{1}{\sqrt{n}} e_{\sigma_{n}}, \lambda\right)$ is the standard normal distribution with mean 0 and variance 1 . Thus by Corollary 1 we see that $\phi$ is not continuous on $\mathfrak{X}$. Since for any $q$-weak distribution on $\mathfrak{X}$ the family of associated distribution functions satisfies the Kolmogorov compatibility conditions we can construct a stochastic process in $R^{x}$ ( $R$ is the real number system) which induces the given distribution functions. If we put $\Omega=R^{*}$ then we can denote this stochastic process by $L(x, \omega)=\omega(x)$ and the joint distribution of $L\left(x_{1}, \omega\right), \cdots, L\left(x_{n}, \omega\right)$ is given by $F_{n}\left(x_{1}, \lambda_{1} ; \cdots ; x_{n}, \lambda_{n}\right)$. See [2]. Taking into account condition (3) of Definition 2.1 it is clear that one should expect the sample functions $L(\cdot, \omega)$ to be linear in some sense. The next theorem states that $L(\cdot, \omega)$ is a linear function for almost all $\omega$.

THEOREM 2. Given a q-weak distribution $L$ on $\mathfrak{X}$ then the stochastic process $L(x, \omega)$ can be realized in the space of all linear functions from $\mathfrak{X}$ to $R$, that is, in the algebraic dual of $\mathfrak{X}$.

Proof. Let $\Omega$ be the set of all linear functions from $\mathfrak{X}$ to $R$, let $\mathfrak{F}$ be the field of cylinder sets of $\Omega . \quad \mathfrak{A} \in \mathfrak{F}$ if and only if $\mathfrak{A}=\left\{\omega /\left(\omega\left(x_{1}\right)\right.\right.$, $\left.\left.\cdots, \omega\left(x_{n}\right)\right) \in A_{n}\right\}$ where $A_{n}$ is a Borel set in $R^{n}$. Let $P_{n}$ be the $n$-dimensional measure induced by $F_{n}\left(x_{1}, \lambda_{1} ; \cdots ; x_{n}, \lambda_{n}\right)$ and then we put $P(\mathfrak{U})=$ $P_{n}\left(A_{n}\right)$. We will now show that $P$ is a completely additive measure on $\mathfrak{F}$.

(1) If $\mathfrak{A} \in \mathfrak{F}$ then $P(\mathfrak{U})$ is uniquely determined. This is proved in exactly the same way as in Kolmogorov [2].

(2) $P(\Omega)=1$. Clear.

(3) If $\mathfrak{A}$ and $\mathfrak{B}$ are disjoint cylinder sets then $P(\mathfrak{A} \cup \mathfrak{B})=P(\mathfrak{A})+P(\mathfrak{B})$.

Let $\mathfrak{U}=\left\{\omega /\left(\omega\left(x_{1}\right), \cdots, \omega\left(x_{k}\right)\right) \in A_{k}\right\}$ and $\mathfrak{B}=\left\{\omega /\left(\omega\left(x_{1}^{\prime}\right), \cdots, \omega\left(x_{j}^{\prime}\right)\right) \in B_{j}\right\}$, then by assumption $\mathfrak{A} \cap \mathfrak{B}=0$. Let $\left(y_{1}, \cdots, y_{n}\right)$ contain all the $x_{i}$ 's and $x_{i}^{\prime}$ 's in some order and let $A$ be the cylinder set in $R^{n}$ with base $A_{k}$ in $R^{k}$ and $B$ be the cylinder set in $R^{n}$ with base $B_{j}$ in $R^{j}$. We claim that $P_{n}(A \cap B)=0$. Suppose not, that is, $P_{n}(A \cap B)>0$. We distinguish two 
cases. First suppose $\left(y_{1}, \cdots, y_{n}\right)$ are linearly independent. $A \cap B \neq 0$ since $P_{n}(A \cap B)>0$. Let $\left(\lambda_{1}, \cdots, \lambda_{n}\right) \in A \cap B$, define $\omega\left(y_{i}\right)=\lambda_{i}$ and extend $\omega$ linearly to the linear extension, $\left\{y_{1}, \cdots, y_{n}\right\}_{L}$, of $\left(y_{1}, \cdots, y_{n}\right)$. Then we can extend $\omega$ to a linear function on all of $\mathfrak{X}$ ( $\omega$ can even be taken to be continuous by the Hahn-Banach theorem). Thus $\omega \in \Omega$ and $\omega \in \mathfrak{U} \cap \mathfrak{B}$ which is a contradiction. Second suppose there is a linear relation, $\sum_{1}^{n} \alpha_{i} y_{i}=0$, among the $y_{i}^{\prime}$ 's. Since

$$
\int_{\sum \alpha_{i} \lambda_{i} \leqq \lambda} d F\left(y_{1}, \lambda_{1} ; \cdots ; y_{n}, \lambda_{n}\right)=\varepsilon(\lambda)
$$

the measure $P_{n}$ in $R^{n}$ is concentrated on the subspace $\sum_{i=1}^{n} \alpha_{i} \lambda_{i}=0$. Because $P_{n}(A \cap B)>0$ there exists a point $\left(\lambda_{1}, \cdots, \lambda_{n}\right) \in A \cap B$ such that $\sum_{i=1}^{n} \alpha_{i} \lambda_{i}=0$. If we define $\omega$ as before we obtain the same contradiction. Thus $P_{n}(A \cap B)=0$. Now

$$
P(\mathfrak{A} \cup \mathfrak{B})=P_{n}(A \cup B)=P_{n}(A)+P_{n}(B)-P_{n}(A \cap B)=P(\mathfrak{A})+P(\mathfrak{B}) .
$$

(4) $P$ is completely additive on $\mathfrak{F}$. This again can be proved exactly as in [2].

We can now extend $P$ to a completely additive measure on the $\sigma$ algebra, $\mathfrak{F}^{\prime}$, generated by $\mathfrak{F}$ and thus the proof of Theorem 2 is complete.

The next theorem gives conditions under which $L(\cdot, \omega)$ is continuous for almost all $\omega$, that is, $L(\cdot, \omega) \in \mathfrak{X}^{*}$ for almost all $\omega$. The proof is fashioned after a proof given by Mann [3] in the real valued case.

THEOREM 3. A necessary and sufficient condition that $L(x, \omega)$ is realizable in the space, $\mathfrak{X}^{*}$, of all continuous linear functions from $\mathfrak{X}$ to $R$ is that for any separable subspace $\mathfrak{X}^{\prime}$ and any $\varepsilon, \eta>0$ there exists $\delta=\delta\left(\varepsilon, \eta, \mathfrak{X}^{\prime}\right)$ such that for any finite collection $x_{1}, \cdots, x_{n} \in \mathfrak{X}^{\prime}$ with $\left\|x_{i}\right\| \leqq \delta$ we have

$$
\int_{-\varepsilon}^{\varepsilon} \cdots \int_{-\varepsilon}^{\varepsilon} d F_{n}\left(x_{1}, \lambda_{1} ; \cdots ; x_{n}, \lambda_{n}\right) \geqq 1-\eta
$$

Proof of sufficiency. First note that if $\lambda_{i}=\varepsilon$ and $\lambda_{i}=-\varepsilon(i=1,2$, $\cdots, n)$ are continuity points of $F_{n}$ then the integral (3.4) is equal to $P\left[\max _{1 \leqq i \leqq n}\left|L\left(x_{i}\right)\right| \leqq \varepsilon\right]$. For the purposes of this proof we denote $\mathfrak{X}^{*}$ by $\Omega$ and then as in the proof of Theorem 2 we can introduce a finitely additive measure, $P$, on the field, $\mathfrak{F}$, of cylinder sets in $\Omega$. We will now show that $P$ is completely additive. As is well known it is sufficient to show that if $\mathfrak{A}_{1} \supset \mathfrak{A}_{2} \supset \cdots$ is a decreasing sequence of cylinder sets 
such that $\bigcap_{n=1}^{\infty} \mathfrak{A}_{n}=0$ then $P\left(\mathfrak{A}_{n}\right) \searrow 0$. Assume $P\left(\mathfrak{A}_{n}\right) \searrow \theta>0$. It is no loss of generality to assume that $\mathfrak{A}_{n}$ is defined by $x_{1}, \cdots, x_{n}$ and a closed Borel set $A_{n}$ in $R^{n}$

$$
\mathfrak{A}_{n}=\left\{\omega \mid\left(\omega\left(x_{1}\right), \cdots, \omega\left(x_{n}\right)\right) \in A_{n}\right\} .
$$

Let $\mathfrak{X}^{\prime}$ be the separable subspace generated by $\left\{x_{1}, x_{2}, \cdots\right\}$, and let $\left\{y_{1}, \cdots\right\}$ be the set of all finite linear combinations of the $x_{i}$ 's with rational coefficients. Thus $\left\{y_{i}\right\}$ is a countable dense set in $\mathfrak{X}^{\prime}$ and we arrange the notation so that $\mathfrak{A}_{n}$ depends on $y_{k_{1}}, \cdots, y_{k_{n}}$ where $k_{i}<k_{j}$ if $i<j$ and $k_{n} \rightarrow \infty$ as $n \rightarrow \infty$. By hypothesis we can choose a $\delta_{2}>0$, independent of $n$, such that

$$
P\left[\omega|\max | \omega\left(y_{i}\right) \mid \leqq \frac{\theta}{4} \text { for } i \leqq k_{n} \text { and }\left\|y_{i}\right\| \leqq \delta_{2}\right] \geqq 1-\frac{\theta}{4} .
$$

Define $\mathfrak{U}_{n}^{2}=\left\{\omega \mid \omega \in \mathfrak{A}_{n}\right.$ and $\left.\max \left|\omega\left(y_{i}\right)\right| \leqq \frac{\theta}{4} ; i \leqq k_{n},\left\|y_{i}\right\| \leqq \delta_{2}\right\}$, then since $P\left(\mathfrak{A}_{n}\right) \geqq \theta$ we have $P\left(\mathfrak{A}_{n}^{2}\right) \geq \frac{\theta}{2}+\frac{\theta}{4}$ and $\mathfrak{A}_{n}^{2} \subset \mathfrak{A}_{n}$. Also $\mathfrak{A}_{1}^{2} \supset \mathfrak{A}_{2}^{2} \cdots$. Similarly we define inductively

$$
\mathfrak{U}_{n}^{p}=\left\{\omega \subseteq \mathfrak{A}_{n}^{p-1}, \max \left|\omega\left(y_{i}\right)\right| \leqq \frac{\theta}{2^{p}} \text { for } i \leqq k_{n},\left\|y_{i}\right\| \leqq \delta_{p}\right\}
$$

and $P\left(\mathfrak{U}_{n}^{p}\right) \geqq \frac{\theta}{2}+\frac{\theta}{2^{p}}$. More over $\mathfrak{U}_{1}^{p} \supset \mathfrak{U}_{2}^{p} \supset \cdots$, and $\mathfrak{U}_{n}^{p} \subset \mathfrak{A}_{n}^{p-1} \subset \cdots \subset \mathfrak{A}_{n}$. Consider the sequence $\mathfrak{U}_{p}^{p}$ and note that $\mathfrak{U}_{1}^{1} \supset \mathfrak{A}_{2}^{2} \supset \cdots$, also note that $\mathfrak{U}_{p}^{p}$ depends on $y_{1}, \cdots, y_{k_{p}}$. Moreover the above inequality shows that $P\left(\mathfrak{A}_{p}^{p}\right) \geq \frac{\theta}{2}$. We can now replace the $\mathfrak{A}_{p}^{p}$ by sets $\mathfrak{B}_{p}$ depending on $y_{1}$, $\cdots, y_{k_{p}}$ such that $\mathfrak{B}_{p} \subset \mathfrak{U}_{p}^{p}$ and the corresponding Borel set, $B_{p}$, in $R^{p}$ is closed and bounded and $P\left(\mathfrak{B}_{p}\right) \geqq \frac{\theta}{4}$. See [2].

Now choose $\omega_{p} \in \mathfrak{B}_{p}$ and by the diagonal process we can choose a subsequence (which we again denote by $\omega_{p}$ ) such that $\omega_{p}\left(y_{i}\right) \rightarrow \lambda_{i}$ for each $y_{i}$. Since $B_{p}$ is closed the point $\left(\lambda_{1}, \cdots, \lambda_{k_{p}}\right)$ is in $B_{p}$ and thus if we define $\omega\left(y_{i}\right)=\lambda_{i}$ for all $y_{i}$ we see that if we can show $\omega \in \mathfrak{X}^{*}$ it will then follow that $\omega \in \mathfrak{B}_{p}$. Clearly $\omega$ is rational linear on $\left\{y_{i}\right\}$. We now show that $\omega$ is uniformly continuous on $\left\{y_{i}\right\}$. Given $\varepsilon>0$ choose $j$ such that $\frac{\theta}{2^{j}} \leqq \frac{\varepsilon}{2}$ and then choose $\delta=\delta_{j}$ (the $\delta_{j}$ used in the construction of $\left.\mathfrak{A}_{n}^{i}\right)$. For any $y_{i}$ with $\left\|y_{i}\right\| \leqq \delta$ we have

$$
\left|\omega\left(y_{i}\right)\right| \leqq\left|\omega\left(y_{i}\right)-\omega_{p}\left(y_{i}\right)\right|+\left|\omega_{p}\left(y_{i}\right)\right|
$$


Since $\omega_{p}\left(y_{i}\right) \rightarrow \omega\left(y_{i}\right)$ we can choose a $p_{0}$ such that $p \geqq p_{0}$ implies $\left|\omega\left(y_{i}\right)-\omega_{p}\left(y_{i}\right)\right| \leqq \frac{\varepsilon}{2}$. We choose a $p$ such that $p \geqq p_{0}, p \geqq j$, and $k_{p} \geqq i$, then $\omega_{p} \in \mathfrak{B}_{p} \subset \mathfrak{A}_{p}^{p} \subset \mathfrak{A}_{p}^{j}$ end hence $\left|\omega_{p}\left(y_{i}\right)\right| \leqq \frac{\theta}{2^{j}} \leqq \frac{\varepsilon}{2}$. Thus if $\left\|y_{i}\right\| \leqq \delta$ then $\left|\omega\left(y_{i}\right)\right| \leqq \varepsilon$. Since $\omega$ is rational linear on $\left\{y_{i}\right\}$ it follows that $\omega$ is uniformly continuous and hence can be extended by continuity to $\mathfrak{X}^{\prime}$. Clearly the extension will be linear on $\mathfrak{X}^{\prime}$, and hence by the Hahn-Banach theorem $\omega$ can be extended to be a continuous linear function on $\mathfrak{X}$. It now follows that $\omega \in \mathfrak{B}_{p}$ for all $p$ and since $\mathfrak{B}_{p} \subset \mathfrak{A}_{p}$ we have that $\omega \in \bigcap_{k=1}^{\infty} \mathfrak{A}_{k}$. Hence $P$ is completely additive on $\mathfrak{F}$.

Proof of necessity. Since $L(\cdot, \omega) \in \mathfrak{X}^{*}$ for almost all $\omega$ we can write $L(x, \omega)=\left\langle x, X^{*}(\omega)\right\rangle$. Let $\mathfrak{X}^{\prime}$ be a separable subspace of $\mathfrak{X}$ and let $\left\|X^{*}(\omega)\right\|^{\prime}$ be the norm of $X^{*}(\omega)$ when considered as a linear functional on $\mathfrak{X}^{\prime}$, then $\left\|X^{*}(\omega)\right\|^{\prime}$ is a measurable function. Given $\varepsilon, \eta>0$ we can choose $\delta>0$ such that $P\left[\left\|X^{*}\right\|^{\prime} \leqq \varepsilon / \delta\right] \geqq 1-\eta$ and this $\delta$ has the required properties.

4. Application to $\mathfrak{X}$ valued random variables. We can now give a solution to the problem stated in the introduction in case $\mathfrak{X}$ is a reflexive space.

Theorem 4. Let $\mathfrak{X}$ be a real reflexive Banach space and $\phi\left(x^{*}\right)$ be a positive definite function on $\mathfrak{X}^{*}$. A necessary and sufficient condition that $\phi$ is the characteristic function of an $\mathfrak{X}$ valued random variable is that:

(i) $\phi(0)=1$ and $\phi$ be continuous on each finite dimensional subspace of $\mathfrak{X}^{*}$.

(ii) If $L=\mathfrak{F}(\phi)$ (which exists by (i) and Theorem 1) then for any separable subspace $\mathfrak{X}_{1}^{*}$ of $\mathfrak{X}^{*}$ and any $\varepsilon, \eta>0$ there exists $\delta=\delta\left(\mathfrak{X}_{1}^{*}, \varepsilon, \eta\right)$ such that for any finite collection $x_{1}^{*}, \cdots, x_{n}^{*} \in \mathfrak{X}_{1}^{*}$ with $\left\|x_{i}^{*}\right\| \leqq \delta$ we have

$$
\int_{-\varepsilon}^{\varepsilon} \cdots \int_{-\varepsilon}^{\varepsilon} d F_{n}\left(x_{1}^{*}, \lambda_{1} ; \cdots ; x_{n}^{*}, \lambda_{n}\right) \geqq 1-\eta \text {. }
$$

Proof. $L$ is a $q$-weak distribution on $\mathfrak{X}^{*}$ which satisfies the conditions of Theorem 3 relative to $\mathfrak{X}^{*}$. Hence $L$ can be realized in $\mathfrak{X}^{* *}=\mathfrak{X}$ since $\mathfrak{X}$ is assumed reflexive. Thus $L\left(x^{*}, \omega\right)=\left\langle x^{*}, X(\omega)\right\rangle$ and $X(\omega)$ is weakly measurable since $L\left(x^{*}, \cdot\right)$ is measurable for all $x^{*}$. But $\phi$ is the characteristic function of $L$ and hence as remarked in $\S 2$ it is the characteristic function of $X$. The necessity of the above conditions is obvious if we apply Theorem 3 . 
We conclude by giving two "continuity" theorems. Suppose $\phi_{n}=$ $\mathfrak{F}\left(X_{n}\right)\left(\mathfrak{F}\left(X_{n}\right)\right.$ denotes the characteristic function of $\left.X_{n}\right)$ and $\phi_{n}\left(x^{*}\right) \rightarrow$ $\phi\left(x^{*}\right)$. Clearly $\phi$ is positive definite and if $\phi$ is continuous at 0 on each finite dimensional subspace then there exists a weak distribution $L$ on $\mathfrak{X}$ such that $\phi=\mathfrak{F}(L)$. The question naturally arises as to when there exists an $X$ such that $\phi=\mathfrak{F}(X)$. We give two theorems which bear on this question and then two examples.

Theorem 5. Let $\mathfrak{X}$ be a real reflexive Banach space and let $\phi_{n}=$ $\mathfrak{F}\left(X_{n}\right)$, if $\phi_{n}\left(x^{*}\right) \rightarrow \phi\left(x^{*}\right)$ then a necessary and sufficient condition that there exist an $X$ such that $\phi=\mathfrak{F}(X)$ is that:

(1) $\phi$ restricted to any finite dimensional subspace of $\mathfrak{X}^{*}$ is continuous at 0 .

(2) Given any separable subspace $\mathfrak{X}_{1}^{*}$ of $\mathfrak{X}^{*}$ and any $\varepsilon, \eta>0$ there exists $a \delta$ such that for any finite collection $J=\left(x_{1}^{*}, \cdots, x_{k}^{*}\right) \in \mathfrak{X}_{1}^{*}$ with $\left\|x_{i}^{*}\right\| \leqq \delta$ there exists $n(J, \delta)$ such that if $n>n(J, \delta)$ then

$$
P\left[\max _{J}\left|L_{n}\left(x_{i}^{*}\right)\right| \leqq \varepsilon\right] \geq 1-\eta .
$$

Proof. Recall that $L_{n}\left(x^{*}\right)=\left\langle x^{*}, X_{n}(\omega)\right\rangle$. We now prove the sufficiency. Condition (1) implies that there exists a weak distribution $L$ such that $\phi=\mathfrak{F}(L)$ and $L_{n} \rightarrow L$ in the sense that

$$
F_{k}^{(n)}\left(x_{1}^{*}, \lambda_{1} ; \cdots ; x_{k}^{*}, \lambda_{k}\right) \rightarrow F_{k}\left(x_{1}^{*}, \lambda_{1} ; \cdots ; x_{k}^{*}, \lambda_{k}\right)
$$

provided $\left(\lambda_{1}, \cdots, \lambda_{k}\right)$ is a continuity point of $F_{k}$. We show that $L$ satisfies the conditions of Theorem 4. For convenience we put $\bar{F}(\varepsilon)=$ $F(\varepsilon, \cdots, \varepsilon)-F(-\varepsilon, \cdots,-\varepsilon)$ for any distribution function $F$ and we say $\varepsilon$ is a continuity point provided $(\varepsilon, \cdots, \varepsilon)$ and $(-\varepsilon, \cdots,-\varepsilon)$ are continuity points of $F$.

Given $\mathfrak{X}_{1}^{*}, \varepsilon, \eta$, choose $\delta$ of Condition (2) corresponding to $\mathfrak{X}_{1}^{*}, \frac{\varepsilon}{2}, \frac{\eta}{2}$, Given any finite collection $J=\left(x_{1}^{*}, \cdots, x_{k}^{*}\right) \in \mathfrak{X}_{1}^{*}$ with $\left\|x_{i}^{*}\right\| \leq \delta$ we must show that

$$
\int_{-\varepsilon}^{\varepsilon} \cdots \int_{-\varepsilon}^{\varepsilon} d F_{k}\left(x_{1}^{*}, \lambda_{1} ; \cdots ; x_{k}^{*}, \lambda_{k}\right) \geqq 1-\eta
$$

Choose $\varepsilon^{\prime}$ such that $\varepsilon^{\prime}$ is a continuity point of $F_{k}$ and $\frac{\varepsilon}{2}<\varepsilon^{\prime}<\varepsilon$. Choose $n_{0} \quad$ such that $n_{0}>n(J, \delta)$ and $\left|\bar{F}_{k}^{n}\left(\varepsilon^{\prime}\right)-\bar{F}_{k}\left(\varepsilon^{\prime}\right)\right| \leqq \frac{\eta}{4}$ then $\bar{F}_{k}\left(\varepsilon^{\prime}\right) \geqq \bar{F}_{k}^{n}\left(\varepsilon^{\prime}\right)-\frac{\eta}{4}$. Since $\varepsilon>\varepsilon^{\prime}$ we have 


$$
\begin{aligned}
\int_{-\varepsilon}^{\varepsilon} \cdots \int_{-\varepsilon}^{\varepsilon} d F_{k}\left(x_{1}^{*}, \lambda_{1} ; \cdots ; x_{k}^{*}, \lambda_{k}\right) & \geqq \bar{F}_{k}\left(\varepsilon^{\prime}\right) \geqq \bar{F}_{k}^{n}\left(\varepsilon^{\prime}\right)-\frac{\eta}{4} \\
& \geq 1-\frac{\eta}{2}-\frac{\eta}{4} \geqq 1-\eta .
\end{aligned}
$$

The necessity is proved by a similar computation.

THEOREM 6. Let $\mathfrak{X}$ be a real separable reflexive Banach space and let $\phi_{n}=\mathfrak{F}\left(X_{n}\right)$, if $\phi_{n}\left(x^{*}\right) \rightarrow \phi\left(x^{*}\right)$ then a sufficient condition that there exist an $X$ such that $\phi=\mathfrak{F}(X)$ is that:

(1) Condition (1) of Theorem 5 hold.

(2) If $G_{n}(a)=P\left[\left\|X_{n}\right\| \leqq a\right]$ then there exists a subsequence $G_{n_{j}}(a)$ converging to a distribution function $G(a)$. ( $\left\|X_{n}\right\|$ is measurable since $\mathfrak{X}$ is separable.)

Proof. In the same way as in the proof of Theorem 5 we have that $L_{n} \rightarrow L$ where $L=\mathfrak{F}(\phi)$ and $L_{n}\left(x^{*}\right)=\left\langle x^{*}, X_{n}\right\rangle$. Given $\left.\varepsilon, \eta\right\rangle 0$ choose $\delta>0$ such that $\frac{\varepsilon}{2 \delta}$ is a continuity point of $G$ and $G\left(\frac{\varepsilon}{2 \delta}\right)>1-\frac{\eta}{2}$. Choose $N$ such that $n_{j}>N$ implies

$$
\left|G_{n_{j}}\left(\frac{\varepsilon}{2 \delta}\right)-G\left(\frac{\varepsilon}{2 \delta}\right)\right|<\frac{\eta}{4} .
$$

Now let $J=\left(x_{1}^{*}, \cdots, x_{k}^{*}\right)$ where $\left\|x_{i}^{*}\right\| \leqq \delta$, and let $\varepsilon^{\prime}$ be a continuity point of $F_{k}\left(x_{1}^{*}, \lambda_{1} ; \cdots, x_{k}^{*}, \lambda_{k}\right)$ such that $\frac{\varepsilon}{2}<\varepsilon^{\prime}<\varepsilon$. (We use same notation as in proof of Theorem 5.) Choose $n_{j}>N$ such that

$$
\left|\bar{F}_{k}^{n} j\left(\varepsilon^{\prime}\right)-\bar{F}_{k}\left(\varepsilon^{\prime}\right)\right|<\frac{\eta}{4}
$$

We now have

$$
\bar{F}_{k}(\varepsilon) \geqq \bar{F}_{k}\left(\varepsilon^{\prime}\right) \geqq \bar{F}_{k}^{n} j\left(\varepsilon^{\prime}\right)-\frac{\dot{\eta}}{4} \geqq \bar{F}_{k}^{n} j\left(\frac{\varepsilon}{2}\right)-\frac{\eta}{4} .
$$

But

$$
\bar{F}_{k}^{n}\left(\frac{\varepsilon}{2}\right)=P\left[\max _{J}\left|\left\langle x_{i}^{*}, X_{n_{j}}\right\rangle\right| \leqq \frac{\varepsilon}{2}\right] \geqq P\left[\delta \cdot\left\|X_{n_{j}}\right\| \leqq \frac{\varepsilon}{2}\right]=G_{n_{j}}\left(\frac{\varepsilon}{2 \delta}\right)
$$

Therefore we finally obtain

$$
\begin{aligned}
\int_{-\varepsilon}^{\varepsilon} \cdots \int_{-\varepsilon}^{\varepsilon} d F_{k}\left(x_{1}^{*}, \lambda_{1} ; \cdots ; x_{k}^{*}, \lambda_{k}\right)=\bar{F}_{k}(\varepsilon) & \geq G\left(\frac{\varepsilon}{2 \delta}\right)-\frac{\eta}{2} \\
& \geq 1-\frac{\eta}{2}-\frac{\eta}{2}=1-\eta,
\end{aligned}
$$


and hence the proof of Theorem 6 is complete.

Corollary 1. Condition (2) of Theorem 6 is implied by

$$
\lim _{a \rightarrow \infty} \inf _{n} G_{n}(a)=1 \text {. }
$$

Proof. In this case every convergent subsequence (at least one exists by the Helly theorem) converges to a distribution function.

Corollary 2. (Mourier [4]). The following condition implies (2) of Theorem 6. For some $\alpha>0 E\left(\left\|X_{n}\right\|^{\alpha}\right)$ exists for all $n$ and $E\left(\left\|X_{n}\right\|^{\alpha}\right) \leqq M$.

\section{Proof. An immediate consequence of Corollary 1.}

ExAMPLE. Let $\mathfrak{X}$ be a separable Hilbert space and let $\left\{e_{n}\right\}$ be a complete orthonormal system. Let $Y_{n}$ be ordinary random variables mutually independent with normal distributions such that $E\left(Y_{n}\right)=0$, $E\left(Y_{n}^{2}\right)=\frac{1}{n}$. Define $X_{n}=\sum_{1}^{n} Y_{k} e_{k}$, clearly $X_{n}$ is an $\mathfrak{X}$ valued random variable. Moreover (identifying $\mathfrak{X}^{*}$ with $\mathfrak{X}$ ), if $x=\sum_{1}^{\infty} \xi_{k} e_{k}$ then

$$
\phi_{n}(x)=E\left\{e^{i \sum_{k=1}^{n} \xi_{k} Y_{k}}\right\}=e^{-1 / 2 \sum_{k=1}^{n} \xi_{k}^{2} / k}
$$

But $\phi_{n}(x) \rightarrow \phi(x)=e^{-1 / 2 \sum_{k=1}^{\infty} \xi_{k}^{2} / k}$ and the convergence is uniform if $\|x\| \leqq A$. Clearly $\phi(x)$ corresponds to the weak distribution $L(x)=\sum_{1}^{\infty} \xi_{k} Y_{k}$. However there is no $\mathfrak{X}$ valued random variable corresponding since $\sum_{1}^{\infty} Y_{k} e_{k}$ diverges with probability one. (This also follows from Theorem 4.) Thus uniform convergence of $\phi_{n}\left(x^{*}\right) \rightarrow \phi\left(x^{*}\right)$ on bounded sets is not sufficient to insure that $\phi$ corresponds to an $\mathfrak{X}$ valued random variable.

ExAmple. This example shows that condition (2) of Theorem 6 is not a necessary condition. Let $\mathfrak{X}$ and $\left\{e_{n}\right\}$ be as in Example 1 . We define an ordinary random variable $Y$ with the following distribution. $P[Y=\sqrt{n}]=P\left[Y^{2}=n\right]=\frac{1}{n !}\left(e^{-\lambda} \lambda^{n}\right)$ for $n=0, \cdots$. Clearly $E(Y) \leqq \lambda$. Let $Y_{n, k}$ be independent random variables each with the above distribution with parameter $\lambda_{n, k}$. Put

$$
\lambda_{n, k}=\left\{\begin{array}{cc}
(1 / n)^{3 / 2} & k \leqq n^{2} \\
0 & k>n^{2}
\end{array} ; \quad \sum_{k=1}^{\infty} \lambda_{n, k}=\sum_{1}^{n^{2}} \lambda_{n, k}=\sqrt{n} .\right.
$$


Let $X_{n}=\sum_{k=1}^{\infty} Y_{n, k} e_{k}=\sum_{k=1}^{n^{2}} Y_{n, k} e_{k}$, then again $X_{n}$ is an $\mathfrak{X}$ valued random variable. If $x=\sum_{k=1}^{\infty} \xi_{k} e_{k}$ then

$$
E\left\{\left|\left(x, X_{n}\right)\right|\right\} \leqq \sum_{k=1}^{n^{2}}\left|\xi_{k}\right| \lambda_{n, k} \leqq\|x\| \cdot\left[\sum_{k=1}^{n^{2}} \lambda_{n, k}^{2}\right]^{1 / 2}=\frac{1}{\sqrt{n}}\|x\| \rightarrow 0 .
$$

Therefore $\left(x, X_{n}\right) \rightarrow 0$ in probability hence $\left(x, X_{n}\right) \rightarrow(x, X)$ in probability where $X \equiv 0$. Thus the weak distributions corresponding to $X_{n}$ approach the weak distribution corresponding to $X$. However $\left\|x_{n}\right\|^{2}=\sum_{k=1}^{n^{2}} Y_{n, k}^{2}$ where the $Y_{n, k}^{2}$ are independent Poisson variables with parameters $\lambda_{n, k}$. Thus the distributions of $\left\|x_{n}\right\|^{2}$ is Poisson with parameter $\sum_{k=1}^{n^{2}} \lambda_{n, k}=\sqrt{n}$ and clearly no subsequence converges to a distribution function.

\section{REFERENCES}

1. S. Bochner, Harmonic analysis and the theory of probability, Berkeley and Los Angeles, 1955.

2. A. N. Kolmogorov, Foundations of the theory of probability, New York, 1950.

3. H. B. Mann, On the realization of stochastic processes by probability distributions in function spaces, Sankhyă, 11, Part 1, (1951), 3-8.

4. E. Mourier, Elements aléatoires dans un espace de Banach, Ann. Inst. H. Poincaré 13 (1953), 161-244.

5. I. E. Segal, Abstract probability spaces and theorem of Kolmogorov, Amer. J. Math. 76 (1954), 721-732.

PRINCETON UNIVERSITY 


\section{PACIFIC JOURNAL OF MATHEMATICS}

EDITORS

H. L. ROYDEN

Stanford University

Stanford, California

R. A. Beaumont

University of Washington

Seattle 5 , Washington
A. R. Whiteman

University of Southern California

Los Angeles 7, California

E. G. Straus

University of California

Los Angeles 24, California

\section{ASSOCIATE EDITORS}
E. F. BECKENBACH
C. E. BURGESS
M. HALL
E. HEWITT
A. HORN
V. GANAPATHY IYER
R. D. JAMES
M. S. KNEBELMAN

L. NACHBIN

I. NIVEN

G. SZEKERES

T. G. OSTROM

M. M. SCHIFFER
F. WOLF

K. YOSIDA

\section{SUPPORTING INSTITUTIONS}

UNIVERSITY OF BRITISH COLUMBIA

CALIFORNIA INSTITUTE OF TECHNOLOGY

UNIVERSITY OF CALIFORNIA

MONTANA STATE UNIVERSITY

UNIVERSITY OF NEVADA

OREGON STATE COLLEGE

UNIVERSITY OF OREGON

UNIVERSITY OF SOUTHERN CALIFORNIA

\author{
STANFORD UNIVERSITY \\ UNIVERSITY OF UTAH \\ WASHINGTON STATE COLLEGE \\ UNIVERSITY OF WASHINGTON \\ AMERICAN MATHEMATICAL SOCIETY \\ CALIFORNIA RESEARCH CORPORATION \\ HUGHES AIRCRAFT COMPANY \\ THE RAMO-WOOLDRIDGE CORPORATION
}




\section{Pacific Journal of Mathematics}

\section{Vol. 7, No. $1 \quad$ January, 1957}

Richard Davis Anderson, Zero-dimensional compact groups of

homeomorphisms ................................... 797

Hans-Joachim Bremermann, Holomorphic functionals and complex

convexity in Banach spaces........................... 811

Hugh D. Brunk, G. M. Ewing and W. R. Utz, Minimizing integrals in

certain classes of monotone functions ................. 833

Philip David, Uniqueness theory for asymptotic expansions in general

regions ...................................... 849

Paul Erdős and Harold Nathaniel Shapiro, On the least primitive root of a

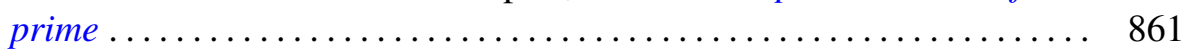

Watson Bryan Fulks, Regular regions for the heat equation ........... 867

William Robert Gaffey, A real inversion formula for a class of bilateral

Laplace transforms ................................ 879

Ronald Kay Getoor, On characteristic functions of Banach space valued random variables ................................. 885

Louis Guttman, Some inequalities between latent roots and minimax (maximin) elements of real matrices ...................... 897

Frank Harary, The number of dissimilar supergraphs of a linear graph .... 903

Edwin Hewitt and Herbert S. Zuckerman, Structure theory for a class of convolution algebras .................................. 913

Amnon Jakimovski, Some Tauberian theorems . . . . . . . . . . . . . . . . . 943

C. T. Rajagopal, Simplified proofs of "Some Tauberian theorems" of Jakimovski................................

Paul Joseph Kelly, A congruence theorem for trees ................. 961

Robert Forbes McNaughton, Jr., On the measure of normal formulas...... 969

Richard Scott Pierce, Distributivity in Boolean algebras .............. 983

Calvin R. Putnam, Continuous spectra and unitary equivalence ......... 993

Marvin Rosenblum, Perturbation of the continuous spectrum and unitary

equivalence................................... 997

V. N. Singh, Certain generalized hypergeometric identities of the

Rogers-Ramanujan type.......................

Peter Swerling, Families of transformations in the function spaces $H^{p} \ldots \ldots 1015$ 